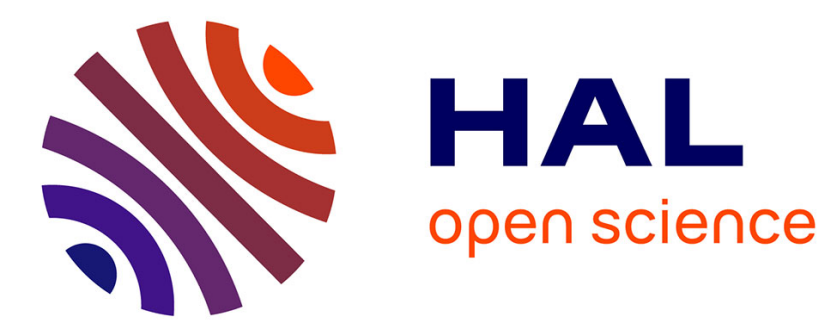

\title{
Snap-through instabilities of pressurized balloons: Pear-shaped bifurcation and localized bulging
}

\author{
T. Wang, F. Xu, Y. Huo, Michel Potier-Ferry
}

\section{To cite this version:}

T. Wang, F. Xu, Y. Huo, Michel Potier-Ferry. Snap-through instabilities of pressurized balloons: Pear-shaped bifurcation and localized bulging. International Journal of Non-Linear Mechanics, 2018, 98, pp.137-144. 10.1016/j.ijnonlinmec.2017.10.017 . hal-01658223

\section{HAL Id: hal-01658223 \\ https://hal.science/hal-01658223}

Submitted on 7 Dec 2017

HAL is a multi-disciplinary open access archive for the deposit and dissemination of scientific research documents, whether they are published or not. The documents may come from teaching and research institutions in France or abroad, or from public or private research centers.
L'archive ouverte pluridisciplinaire HAL, est destinée au dépôt et à la diffusion de documents scientifiques de niveau recherche, publiés ou non, émanant des établissements d'enseignement et de recherche français ou étrangers, des laboratoires publics ou privés. 


\title{
Snap-through instabilities of pressurized balloons: Pear-shaped bifurcation and localized bulging
}

\author{
T. Wang ${ }^{\mathrm{a}}, \mathrm{F} . \mathrm{Xu}^{\mathrm{a}, *}$, Y. Huo ${ }^{\mathrm{a}}$, M. Potier-Ferry ${ }^{\mathrm{b}}$ \\ a Institute of Mechanics and Computational Engineering, Department of Aeronautics and Astronautics, Fudan University, 220 Handan Road, Shanghai 200433, PR China \\ b Laboratoire d'Etude des Microstructures et de Mécanique des Matériaux, LEM3, UMR CNRS 7239, Université de Lorraine, 7 Rue Félix Savart, BP 15082 , 57073 Metz \\ Cedex 03, France
}

This paper investigates numerically the post-bifurcation evolution of ellipsoidal or spherical balloons subject to internal pressure, where the primary -shaped curves of pressure vs. principal stretch and the corresponding bifurcated branch, i.e. pear-shaped deformation, are captured quantitatively. We quantify and discuss the range of pear-shaped bifurcation intervals of ellipsoids and the associated critical points. For rugby-shaped ellipsoidal balloons, we find that there exists a threshold for the aspect ratio of the major and minor axes that leads to pear-shaped bifurcation, which is detected by the finite element method. When the rugby shape becomes sufficiently slender, the nonlinear response of the ellipsoidal balloons is well approximated by the deformation of a tube with localized bulging instead of pear-shaped configuration. We obtain the nonlinear evolution of the localized bulging of rugby-like ellipsoids numerically. Furthermore, we examine the influence of various aspect ratios for rugby-shaped balloons on the localized bulging response. We find that pumpkinshaped ellipsoids can always bifurcate into pear shape. Lastly, we provide a unified phase diagram on instability mode selection of various aspect ratios of ellipsoidal balloons, with diverse representative deformed configurations.
Keywords:

Pressurized balloon

Snap-through instability

Bifurcation

Pear shape

Localized bulging

\section{Introduction}

Three-dimensional elastic membranes such as balloons have various engineering and biomedical applications including parachutes, gas inflated cushion roof panels, air beams, automobile airbags and human brain tissue [1-4]. Nonlinear inflation of pressurized membranes, especially hyperelastic balloons, is a classical finite deformation problem in continuum mechanics, which has received considerable attention in the past few years [5-11]. A basic characteristic of inflated rubber-like balloons is their $N$-shaped snap-through nonlinear finite deformation: the pressure increases sharply at first, then declines and re-rises finally with respect to the increasing principal stretch. Haughton and Ogden [12] derived the membrane equilibrium equations of nonlinear elasticity based on the consistent use of average conjugate variables.

Later, Haughton [13] showed that bifurcation points must be shown in pairs between the maximum and minimum inflated pressure. Precisely, the occurrence of the first bifurcation point corresponds to the initiation of an aspherical non-homogeneous deformation, while the second bifurcation refers to the return to the homogeneous spherical state. Moreover, they established the equilibrium equations governing the axisymmetric inflation of an initial axisymmetric membrane. A number of workers have since obtained the bifurcated solutions through solving these equations by different numerical methods. Chen and Healey [14] used a general class of strain energy functions to model the membrane as a two-dimensional elastomer, and demonstrated the existence of an isola bifurcation which is consistent with experimental observations. Müller and Struchtrup [15] examined an inflation apparatus to model the lung and balloon, and explained the process of the $N$-shaped pressure-radius curve. Fu and Xie [16] made a further study on the basis of Haughton [13] with the numerical shooting method and found that the pear-shaped deformation of spherical balloons is unstable under pressure control while stable under mass control. Beyond the pure mechanical loading, the inflation of balloons under an electromechanical coupling field has been taken into account [17-20]. Liang and Cai [21] conducted a linear perturbation analysis on the spherical dielectric elastomer balloon under the actions of internal pressure and electric voltage, and revealed that the pear-shaped deformation becomes more obvious due to the contribution of electric field.

\footnotetext{
* Corresponding author.

E-mail address: fanxu@fudan.edu.cn (F. Xu).
} 
Lately, Geng et al. [22] extended the work of Fu and Xie [16] to ellipsoidal configurations and found that it is always possible for pumpkinshaped balloon to bifurcate into pear shape, whereas there exists a threshold of aspect ratio for rugby-shaped balloon. When the aspect ratio of the major and minor axes is smaller than a critical value, the inflation behavior of the ellipsoid of revolution is approximated to the response of a tube. For hyperelastic tubes, instead of pear-shaped solutions normally found in balloons, localized bulging deformation is a common nonlinear response [23-28]. Several numerical works based on finite element method, studying the nonlinear response of tube inflation were also conducted [29-31]. The effect of aspect ratio, wall thickness and boundary condition on the $N$-shaped curve were discussed in Shi and Moita [29]. Pamplona et al. [30] and Gonçalves et al. [31] investigated the localized bulging behavior of tubes experimentally and numerically using finite element method, through considering only one term in the Ogden constitutive law [32,33]. Apart from the pure mechanical field, electro-mechanical coupling bifurcation and bulging propagation of the tube were also carried out experimentally and analytically [34,35].

In the literature mentioned above, the post-bifurcation behavior of pressurized balloons was generally pursued analytically or experimentally. Although finite element analysis was performed in several works [29-31,36], the snap-through nonlinear deformation of inflated balloons and its evolution involving both primary and bifurcated branches have not been explored to date and merit further systematic investigation. Therefore, this paper aims to fill this gap in the literature by quantitatively exploring the post-bifurcation evolution of pressurized ellipsoidal/spherical balloons and their sphere/pear/localized mode transitions by using the finite element method. Imperfection sensitivity of the post-bifurcation response, especially the descending bifurcated branch involving mode transitions, is carefully examined. Moreover, a large range of dimensionless aspect ratios of the major and minor axes for ellipsoidal balloons is considered and its influence on the instability mode is discussed. The nonlinear evolution of localized bulging for slender rugby balloons, which is approximated to a tube, has not been studied numerically to the best of our knowledge. Lastly, a general phase diagram involving diverse geometric configurations, e.g. ellipsoids of revolution, spheres and tubes, is drawn to provide a whole view of postbifurcation mode selection.

The paper is organized as follows. In Section 2, a mechanical model of ellipsoidal balloons subject to internal pressure is presented, and then a finite element model and its nonlinear resolution algorithm are given in Section 3. Results and discussions are reported in Section 3, including the comparison of various bifurcation curves and the demonstration of a unified phase diagram on instability mode selection.

\section{Model}

We explore the nonlinear deformation of an ellipsoidal balloon subject to internal pressure $P$. The surface of an ellipsoidal balloon can be formed by revolving an ellipse in the $Z R$-plane around the $Z$ axis, which leads to two types of ellipsoidal balloons (see Fig. 1): rugbyshaped $(a / b<1)$ and pumpkin-shaped $(a / b>1)$ balloons, where $a$ and $b$ denote the semi-axes of the ellipse which form the ellipsoidal balloon by rotating around $Z$ axis. Particularly, the case of $a / b=1$ corresponds to a spherical balloon. We set $\max \{a, b\}$ as a unit in all the examined cases for generality.

The initial undeformed ellipsoidal balloon can be described by

$R(\theta)=a \sin \theta, \quad Z(\theta)=-b \cos \theta, \quad 0 \leqslant \theta \leqslant \pi$.

We focus on axisymmetric deformations which can be expressed as

$r=r(\theta), \quad z=z(\theta), \quad 0 \leqslant \theta \leqslant \pi$,

where $r$ and $z$ are cylindrical polar coordinates in the deformed state with respect to the angle $\theta$. Then, one has

$\mathrm{dS}=\sqrt{(\mathrm{dR})^{2}+(\mathrm{dZ})^{2}}=\chi(\theta) \mathrm{d} \theta, \quad \mathrm{ds}=\sqrt{\left(\frac{\mathrm{dr}}{\mathrm{d} \theta}\right)^{2}+\left(\frac{\mathrm{dz}}{\mathrm{d} \theta}\right)^{2}} \mathrm{~d} \theta$,

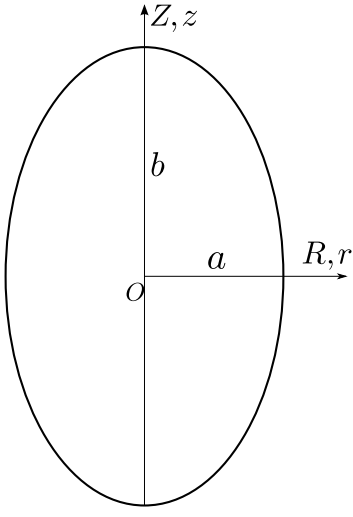

(a)

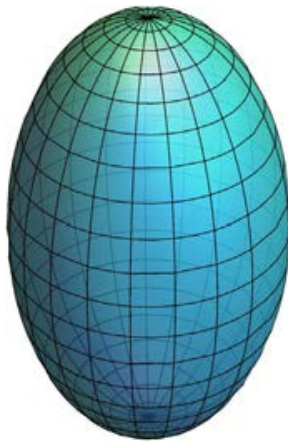

(b)

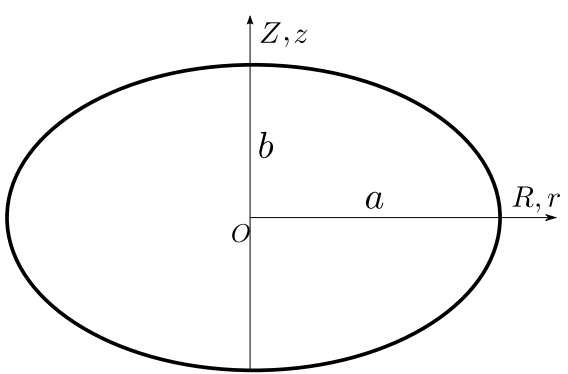

(c)

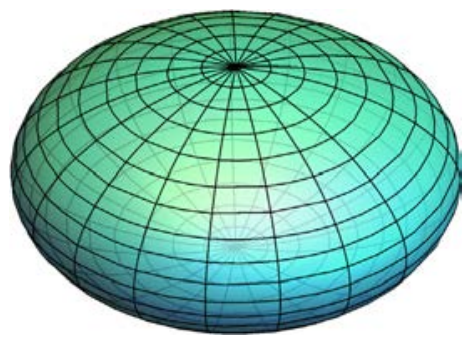

(d)

Fig. 1. Ellipsoidal balloons: (a) rugby-shaped balloon $(a / b<1)$; (b) 3D rugby-shaped balloon in the undeformed state; (c) pumpkin-shaped balloon $(a / b>1)$; (d) 3D pumpkinshaped balloon in the undeformed state.

where $\mathrm{d} S$ and $\mathrm{d} s$ represent, respectively, the reference and current arclengths, in which $\chi(\theta)$ is denoted by

$\chi(\theta)=\sqrt{a^{2} \cos ^{2} \theta+b^{2} \sin ^{2} \theta}$.

The principal stretches are

$\lambda_{1}=\frac{r}{R}, \quad \lambda_{2}=\frac{\mathrm{ds}}{\mathrm{dS}}=\frac{1}{\chi(\theta)} \sqrt{\left(\frac{\mathrm{dr}}{\mathrm{d} \theta}\right)^{2}+\left(\frac{\mathrm{dz}}{\mathrm{d} \theta}\right)^{2}}, \quad \lambda_{3}=\frac{h}{H}$,

where $H$ and $h$ are the thicknesses in the reference and current configurations, respectively. By considering the balance of the infinitesimal volume, the equilibrium equations can be obtained as [13]:

$\left\{\begin{array}{l}r \frac{\mathrm{d}\left(h \sigma_{2}\right)}{\mathrm{dS}}+h \frac{\mathrm{dr}}{\mathrm{dS}}\left(\sigma_{2}-\sigma_{1}\right)=0, \\ \frac{\mathrm{dz}}{\mathrm{dS} \sigma_{1}}+\frac{\sigma_{2}\left(\frac{\mathrm{dr}}{\mathrm{d} \mathrm{S}} \frac{\mathrm{d}^{2} z}{\mathrm{dS}^{2}}-\frac{\mathrm{dz}^{2} \mathrm{dS}}{\mathrm{d}^{2} r}\right)}{\lambda_{2}^{3}}=\lambda_{1} \lambda_{2} \bar{P},\end{array}\right.$

where $\sigma_{1}$ and $\sigma_{2}$ are the principal Cauchy stresses, and $\bar{P}=P / \mu H$, where $\mu$ is the ground state shear modulus. By substituting the relations $\mathrm{dr} / \mathrm{dS}=\lambda_{2} \sin \phi, \mathrm{dz} / \mathrm{dS}=\lambda_{2} \cos \phi$ and the constitutive relations $\sigma_{i}=$ 
$\lambda_{i} W_{i}$ (no summation for $i$ ) $[37,38]$ into Eq. (6), we obtain the following differential equation:

$\frac{\mathrm{d} \phi}{\mathrm{d} \theta}=\frac{W_{1} \chi(\theta) \cos \phi}{W_{2} R}-\frac{\bar{P} \lambda_{1} \lambda_{2} \chi(\theta)}{W_{2}}$,

where $\phi$ is the angle between the meridian and $z$ axis, $W$ denotes the strain energy density function which is expressed as a function of $\lambda_{i}$, and $W_{i}=\partial W / \partial \lambda_{i}, W_{i j}=\partial^{2} W / \partial \lambda_{i} \partial \lambda_{j}(i, j=1,2)$.

With respect to material models, the rubber-like balloon can bifurcate into pear shape when the inflation reaches the critical value for the Ogden material in general, but not for the neo-Hookean, MooneyRivlin or Gent models in the purely mechanical loading case. A thorough discussion on the dependence of bifurcation on the constitutive models was carried out by Xie et al. [39]. For rubber balloons, assuming incompressibility, the three-term Ogden material [32,33] reads

$W=\sum_{i=1}^{3} \tilde{\mu}_{i}\left(\lambda_{1}^{\alpha_{i}}+\lambda_{2}^{\alpha_{i}}+\lambda_{3}^{\alpha_{i}}-3\right) / \alpha_{i}$,

where $\alpha_{i}$ and $\tilde{\mu}_{i}$ are material constants. In this paper, we take the following material parameters from [32], which are widely used for the analyses of inflation of hyperelastic balloons [16,22,27,39]: $\alpha_{1}=1.3$, $\alpha_{2}=5.0, \alpha_{3}=-2.0, \tilde{\mu}_{1}=1.4919, \tilde{\mu}_{2}=0.003$ and $\tilde{\mu}_{3}=-0.024$, in which $\tilde{\mu}_{i}$ are dimensionless, scaled by the ground state shear modulus $\mu$. Practically, these values of the parameters refer to a vulcanized natural rubber which has highly reversible hyperelastic behavior but low crystallization upon extreme stretching [40]. Furthermore, the strain energy density function $W$ and the internal pressure $P$ are nondimensionalized by choosing the ground state shear modulus to be unity.

\subsection{Spherical balloon $(a / b=1)$}

Let us begin by the spherical balloon when $a / b=1$. In cylindrical coordinates, the spherical solution can be obtained by considering

$\lambda_{1}=\lambda_{2}=\lambda, \quad \phi=\frac{\pi}{2}-\theta$,

and the equilibrium equation (6) yields the following relation between $\bar{P}$ and $\lambda$ :

$\bar{P}=\frac{2 W_{2}}{a \lambda^{2}}$,

where $\lambda$ is a constant. This solution refers to a homogeneous inflation and keeps the shape spherical during deformation. Then, the bifurcation condition for a non-uniform deformed configuration [16] is given by

$\frac{\lambda^{2}}{H} \frac{\mathrm{d}\left(h \sigma_{1}\right)}{\mathrm{d} \lambda}=\lambda^{2} \frac{\mathrm{d}}{\mathrm{d} \lambda}\left(\frac{W_{1}}{\lambda}\right)=\lambda\left(W_{11}+W_{12}\right)-W_{1}=0$.

By solving this ordinary differential equation (ODE), we can obtain the critical and limit values of $\lambda$ for the Ogden constitutive law as

$\lambda_{c r 1}=1.7783, \quad \lambda_{c r 2}=2.5137, \quad \lambda_{\max }=1.3742, \quad \lambda_{\min }=4.3237$,

and the corresponding pressures read

$\bar{P}_{c r 1}=1.1036, \quad \bar{P}_{c r 2}=0.7633, \quad \bar{P}_{\max }=1.3017, \quad \bar{P}_{\text {min }}=0.5663$.

The spherical balloon can deform to pear shape when $\lambda$ increases to $\lambda_{c r 1}$, and keeps going until the value reaches $\lambda_{c r 2}$. Beyond $\lambda_{c r 2}$, the pear-shaped balloon will return to the spherical shape. For the inhomogeneous deformation, it is, however, rather difficult to have an explicit form of analytical solutions other than numerical solutions. A shooting method was adopted by $\mathrm{Fu}$ and Xie [16] to numerically solve Eq. (6), while a linear perturbation analysis was performed by Liang and Cai [21] to calculate critical conditions of Eq. (6).

\subsection{Rugby-shaped balloon $(a / b<1)$}

A rugby-shaped balloon refers to non-equal axes with $a / b<1$. In [22], the governing equation (6) is decomposed into three first-order ODEs that can be solved by numerical shooting method. Their results show that the bifurcation interval shortens with the decrease of $a / b$, and then disappears at $a / b=0.626$. This point will be further examined in this work by the finite element method. Below this value, the response of the rugby-shaped balloon would be approximated to a tube that may not bifurcate into pear shape. For tubes, the localized bulging phenomenon normally dominates and has been observed in experiments [30,31].

\subsection{Pumpkin-shaped balloon $(a / b>1)$}

Differing from rugby configuration, for a pumpkin-shaped balloon with $a / b>1$, bifurcated solutions always exist with pear-shaped deformation. In [22], it is, however, difficult to distinguish the bifurcated and primary solutions of pressure-volume curves analytically. Through finite element method, we can clearly detect bifurcated branches from pressure-volume curves.

\section{Finite element results and discussion}

There have been a number of analytical and experimental works on the inflation of balloons as mentioned above, but only few works focused on numerical approaches which can provide an overall view of post-bifurcation evolution. Here we employ the finite element method in the commercial software Abaqus [41] to compute the whole inflation process of various balloons under internal pressure.

It is worth mentioning that to facilitate the numerical description of deformations, the Cartesian coordinate system is applied in finite element models, in which the origin is set at the bottom pole of the balloon, while $x$ and $z$-axis are parallel to $R$ and $Z$-axis, respectively. Since the deformation is generally axisymmetric, one quarter of balloon with suitable symmetric boundary conditions can be considered for the discretization, and the bottom pole of the balloon is fixed to prevent rigid body motions. Note that all the deformation figures in what follows are drawn by mirroring the computational model in two planes. Of course, non-axisymmetric solutions also exist in experiments under sufficient perturbations from geometric imperfections, see [28] for example, which is a topic beyond the scope of the present work.

The symmetric mesh (of the equator and $45^{\circ}$ longitude) throughout the work is based on a gridding technique named "cubed sphere" [42] which can remove the presence of any geometrical singularity at the poles and provide the most similar element sizes to have high accuracy and good convergence. By contrast, non-symmetric meshes may lead to artificial symmetry-breakings and bifurcations of the system. By using the Riks arc-length method, one single bifurcated branch can be detected in the post-bifurcation stage. Other bifurcated solutions may also be obtained [43-45], provided that sufficient perturbations are applied. The membrane element M3D4, which can represent thin surfaces in space and offer stretching strength in the plane without bending stiffness, has been adopted. For thin rubber-like materials that forms a balloon, this membrane element is particularly suitable.

We define how the membrane thickness changes with deformation by specifying a nonzero value for the section Poisson's ratio, which allows for a change in the thickness of the membrane as a function of the in-plane strains in geometrically nonlinear analysis. Throughout the paper, this section Poisson's ratio is set to be 0.5 to enforce the incompressibility of the element. There exists negative stiffness stage on the $N$-shaped pressure-volume curve, where the nonlinear response shows a snap-through instability. Static equilibrium states during the unstable phase of this response can be captured by using the modified Riks method. In Abaqus, the Ogden material is defined as

$\tilde{W}=\sum_{i=1}^{3} \frac{2 \mu_{i}}{\alpha_{i}^{2}}\left(\bar{\lambda}_{1}^{\alpha_{i}}+\bar{\lambda}_{2}^{\alpha_{i}}+\bar{\lambda}_{3}^{\alpha_{i}}-3\right)+\sum_{i=1}^{3} \frac{1}{D_{i}}(J-1)^{2 i}$, 


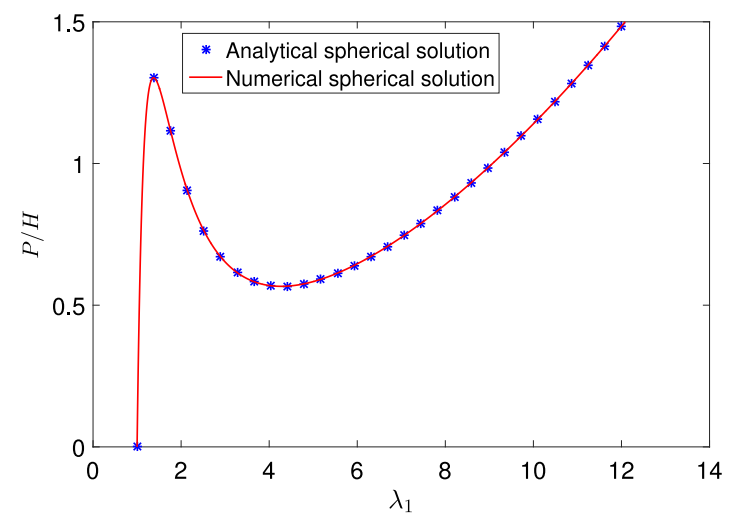

Fig. 2. $N$-shaped diagram of numerical and analytical results when $a / b=1$. The values of FEM/analytical solutions: $\bar{P}_{\max }=1.3025 / 1.3017, \bar{P}_{\min }=0.5666 / 0.5663, \lambda_{\max }=$ $1.3761 / 1.3742$ and $\lambda_{\min }=4.2630 / 4.3237$.

where $\bar{\lambda}_{i}=J^{-1 / 3} \lambda_{i}$, with $J$ being the total volume strain and $J=1$ for incompressible materials, and $D_{i}$ are temperature-dependent material parameters. In particular, the last term does not contribute to the energy function for fully incompressible materials. With the relation of $2 \mu_{i} / \alpha_{i}=$ $\tilde{\mu}_{i}$, the material parameters corresponding to Eq. (8) yield: $\alpha_{1}=1.3$, $\alpha_{2}=5.0, \alpha_{3}=-2.0, \mu_{1}=0.9692, \mu_{2}=0.0075$ and $\mu_{3}=0.024$.

\subsection{Spherical balloon $(a / b=1)$}

We begin by exploring the post-bifurcation of spherical balloons when $a / b=1$. The numerical $N$-shaped curve of $\bar{P}$ and $\lambda_{1}$ matches remarkably with the theoretical spherical solution (10), as shown in Fig. 2. During inflation, $\bar{P}$ grows linearly until it reaches its maximum value $\bar{P}_{\max }=1.3025$ when $\lambda_{1}=1.3761$, then gradually decreases to the minimum $\bar{P}_{\min }=0.5666$ when $\lambda_{1}=4.2630$, and finally increases monotonically. The corresponding analytical values are given in Eqs. (12) and (13), which are highly consistent with the numerical solutions.

As mentioned in Section 2.1, there exist bifurcated solutions in the descending stage during nonlinear deformation. Numerical bifurcated solutions can be obtained by adding an initial imperfection to break the symmetry. The Riks method appears to be sufficiently versatile to trace the bifurcation curve in this problem. In order to trigger a transition from fundamental to bifurcated branches, a fictitious perturbation force is imposed on the membrane. Introduction of such small perturbation forces is a common technique for solving bifurcation problems by continuation techniques $[46,47]$, even when using commercial finite element codes like Abaqus. Here the perturbation force allows computing the whole bifurcated branch with a single continuation algorithm.

For spherical balloons, an initial geometric imperfection $(a / b=$ 0.999 ) is required to break the symmetry of spherical geometry. To distinguish the bifurcation interval, both bifurcated and primary solutions are plotted in Fig. 3, with comparison of analytical bifurcated values from Eqs. (12) and (13). It can be seen that the numerical and theoretical solutions are remarkably consistent. The balloon is homogeneously inflated at the beginning, then suddenly deforms into pear shape (see Fig. 4 where a scaling factor of 100 is taken for illustrative purpose) when $\lambda_{c r 1}=1.7750$, and finally returns to the fundamental uniform expansion beyond $\lambda_{c r 2}=2.5410$. The effect of mesh convergence on post-buckling evolution is further examined in Fig. 5. One can observe that the convergent meshes have no apparent influence either on post-bifurcation evolution or on critical pressures. For the other cases throughout the paper, mesh convergence was carefully checked as well, especially for those with bifurcation paths.

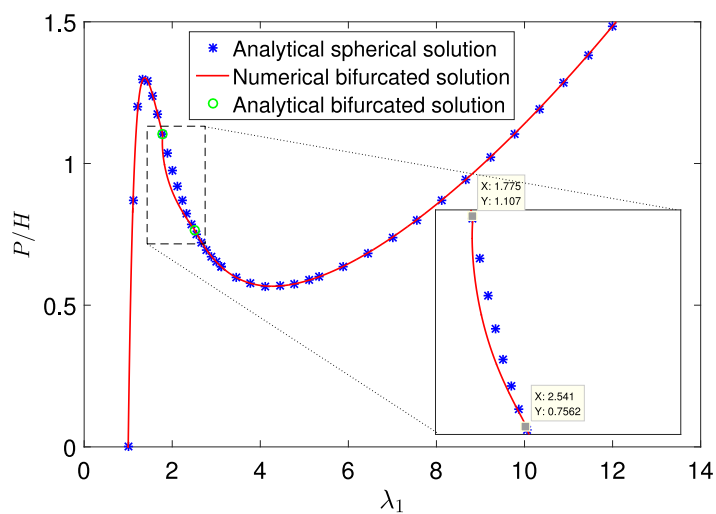

Fig. 3. The analytical spherical, bifurcated and numerical bifurcated solutions: $a / b=1$. The values of FEM/analytical bifurcated critical solutions: $\lambda_{c r 1}=1.7750 / 1.7783, \lambda_{c r 2}=$ 2.5410/2.5137, $\bar{P}_{c r 1}=1.1070 / 1.1036$ and $\bar{P}_{c r 2}=0.7562 / 0.7633$.

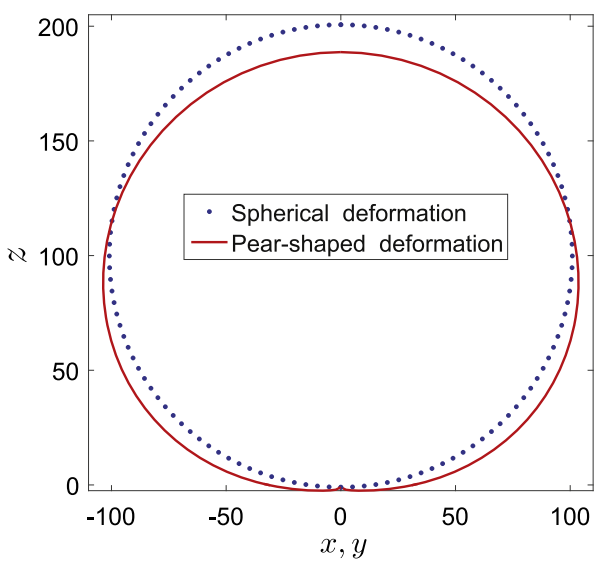

(a)

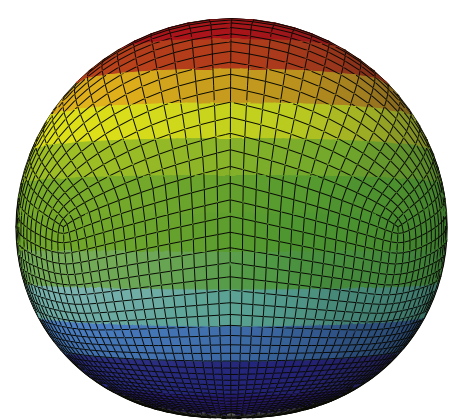

(b)

Fig. 4. Deformation of spherical balloon within the bifurcation interval: (a) cross-section of spherical and pear-shaped deformation; (b) 3D pear-shaped deformation. Scaling factor: 100 .

\subsection{Rugby-shaped balloon $(a / b<1)$}

When the aspect ratio $a / b<1$, we have a rugby-shaped balloon. We explore the effect of a wide range of this ratio on post-bifurcation evolution by starting from $a / b=0.8$. The numerical primary and bifurcated $N$-shaped curves are drawn in Fig. 6 , in which the theoretical bifurcated thresholds [22] are compared. When the scaling volume $\bar{V}=V / \pi$ rises to $\bar{V}_{c r 1}=6.177$, the rugby-shaped balloon can initially bifurcate into pear shape (see Fig. 7) under small perturbations. When $\bar{V}=\bar{V}_{c r 2}=16.41$, the pear-shaped bifurcation will recover the primary path. 


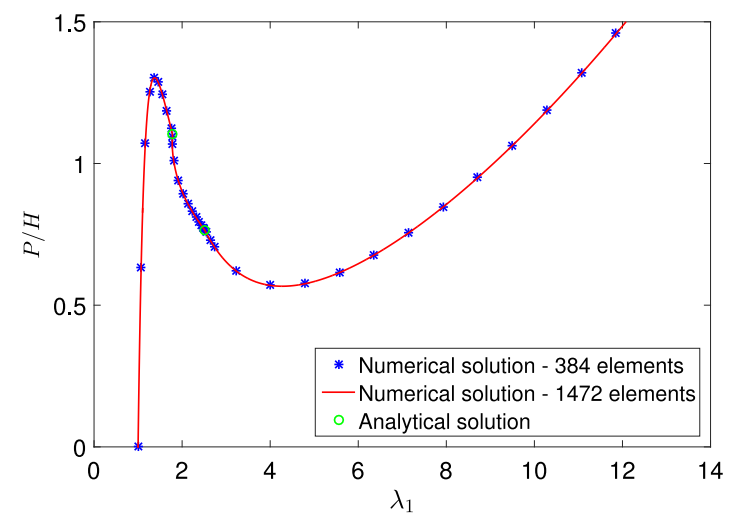

Fig. 5. Mesh convergence test for spherical balloon with $a / b=1$. One can see that 384 elements are sufficient for a convergent solution and more refined mesh has no apparent influence on the bifurcation path as well as critical pressures.

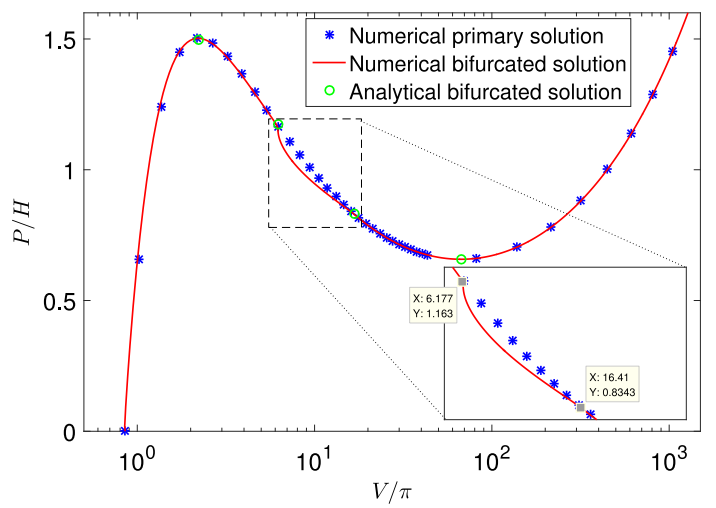

Fig. 6. Bifurcation portrait of ellipsoidal balloon with $a / b=0.8$. The critical bifurcated solutions are: $\bar{V}_{c r 1}=6.177, \bar{V}_{c r 2}=16.41, \bar{P}_{c r 1}=1.163$ and $\bar{P}_{c r 2}=0.8343$, where the scaling volume $\bar{V}=V / \pi$. The limit points read: $\bar{P}_{\max }=1.5024, \bar{P}_{\min }=0.6577, \bar{V}_{\max }=2.206$ and $\bar{V}_{\min }=66.9464$.

Table 1

Finite element results on critical and limit loads for different $a / b$.

\begin{tabular}{lllll}
\hline$a / b$ & $\bar{P}_{\max }$ & $\bar{P}_{c r 1}$ & $\bar{P}_{c r 2}$ & $\bar{P}_{\min }$ \\
\hline 0.8 & 1.5024 & 1.1630 & 0.8343 & 0.6577 \\
0.7 & 1.6326 & 1.1410 & 0.9179 & 0.7206 \\
0.65 & 1.7079 & 1.0960 & 0.9447 & 0.7590 \\
0.63 & 1.7408 & 1.0510 & 0.9937 & 0.7759 \\
\hline
\end{tabular}

The post-bifurcation response of rugby-shaped balloons with decreasing values of $a / b$, i.e. $a / b=0.7,0.65,0.63$ and 0.626 , is investigated. Critical and limit pressures are listed in Table 1 . We note that the bifurcation interval $\left(\bar{P}_{c r 1}-\bar{P}_{c r 2}\right)$ shortens with the declining $a / b$, which can be observed from bifurcation curves in Figs. 6, 8 and 9. When the ratio $a / b$ declines to 0.63 , the bifurcation interval almost vanishes, with $\bar{P}_{c r 1}-\bar{P}_{c r 2}=1.051-0.9937=0.0573$. One can hardly distinguish this interval when $a / b=0.626$ (see Fig. 9) and thus the value can be viewed as a threshold for the disappearance of bifurcated branch, which is consistent with the analytical solution in [22].

With further decreasing of the ratio $a / b$, the rugby-shaped balloon is approaching to a tube where localized bulging deformation instead of pear-shaped bifurcation will appear. For inflation of a tube-like balloon, localized bulging first occurs in the middle, and then gradually propagates to both ends. The whole evolution of localized bulging of a balloon with $a / b=0.1$ is illustrated in Fig. 10. Interestingly, Eriksson and Nordmark [9] studied the instability behavior of a long cylindrical balloon based on Mooney-Rivlin hyperelastic constitutive laws. The tube-like balloon $(a / b=0.1)$ considered here holds qualitatively similar $N$-shaped curve and localized bugling phenomenon to the long

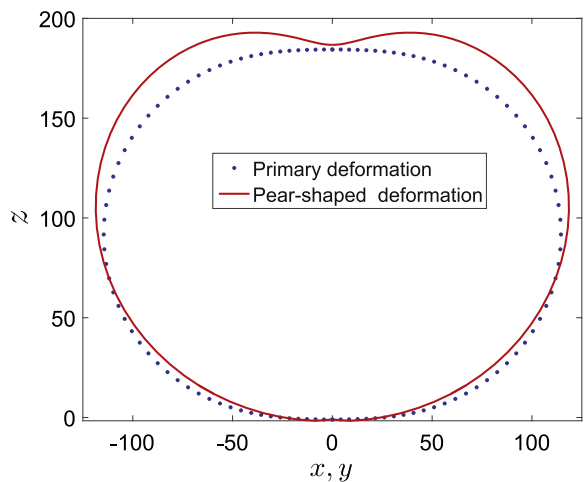

(a)

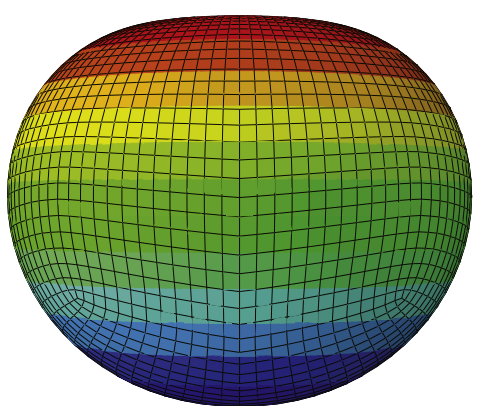

(b)

Fig. 7. Deformation of rugby-shaped balloon with $a / b=0.8$ within the bifurcation interval: (a) cross-section of spherical and pear-shaped deformation; (b) 3D pear-shaped deformation. Scaling factor: 100.

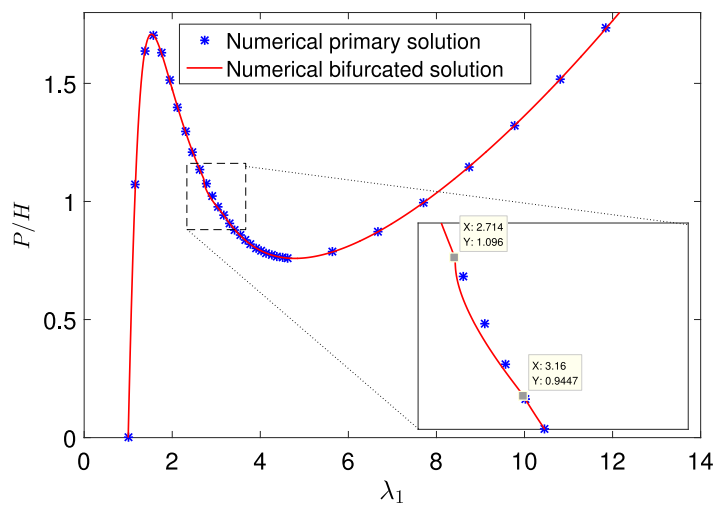

Fig. 8. Bifurcation diagram of primary and bifurcated solutions for rugby-shaped balloons with $a / b=0.65$. The bifurcated solutions: $\lambda_{c r 1}=2.714, \lambda_{c r 2}=3.16, \bar{P}_{c r 1}=1.096$ and $\bar{P}_{c r 2}=0.9447$.

cylindrical balloon, even though the material models are different. It seems that the small curvature along the long axis in tube-like balloons and different material laws have no essential influence on the localized bulging phenomenon qualitatively.

Next we look into the localized bulging evolution of the rugbyshaped balloon $(a / b<0.626)$ in greater depth. To highly approximate to the nonlinear characteristics of a tube, we set $a / b=0.01$. The Point $A$ at the bulging center and Point $B$ at sufficient distance away from the Point $A$ are chosen, respectively, since the localized bulging $\left(\lambda_{1}^{A}\right)$ can be characterized by Point $A$ while the homogeneous deformation $\left(\lambda_{1}^{B}\right)$ can be represented by Point $B$. The relation between $\lambda_{1}^{A}$ and $\lambda_{1}^{B}$ is then plotted in Fig. 11(a). The initial inflation $O \rightarrow C$ corresponds to a uniform deformation. Localized bulging occurs at $C$ and then steadily propagates along $\lambda_{1}^{B}$ from $D$ to $E$. Simultaneously (as shown in 


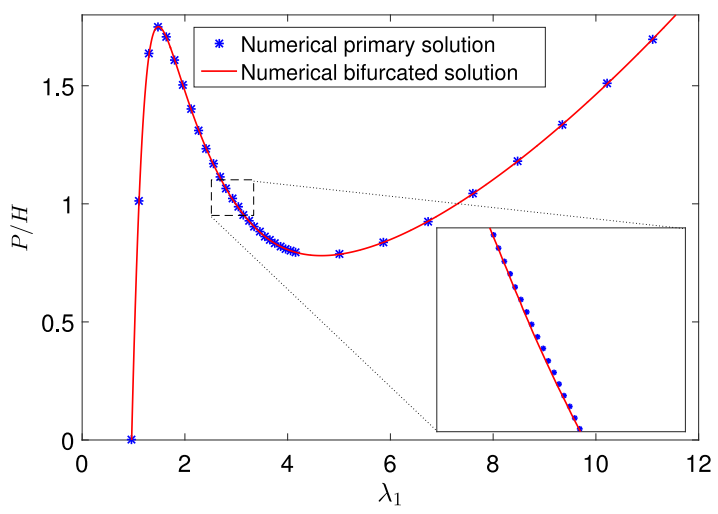

Fig. 9. Bifurcation diagram of primary and bifurcated solutions for rugby-shaped balloons with $a / b=0.626$. No distinguished bifurcation is observed.

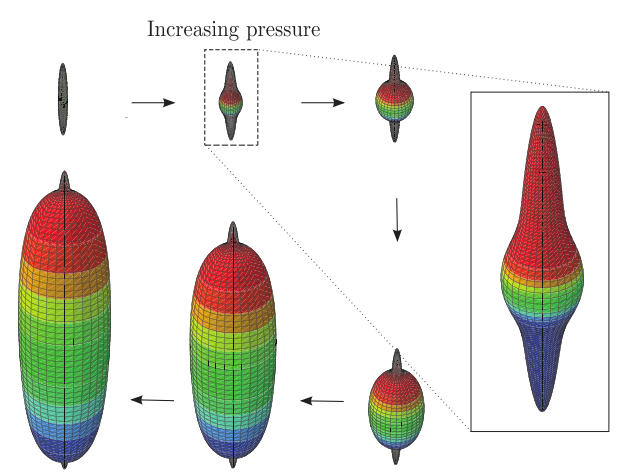

(a)

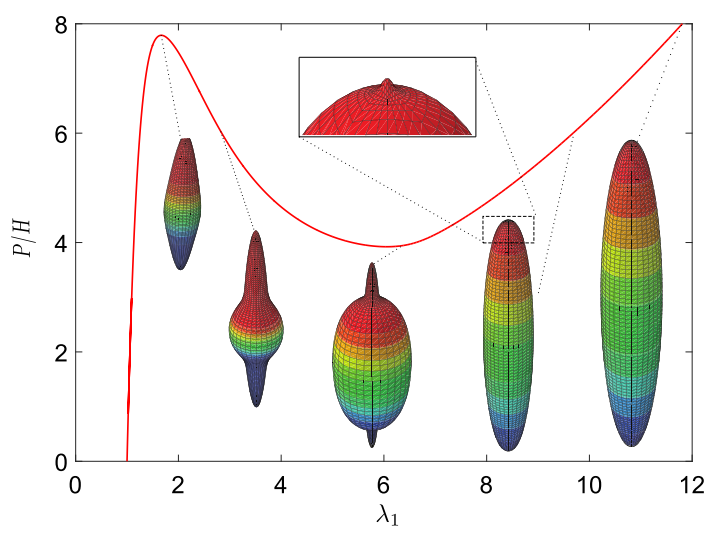

(b)

Fig. 10. Localized bulging of an inflated balloon with $a / b=0.1$ : (a) deformation evolution; (b) pressure-stretch curve.

Fig. 11(b)), in the beginning of the uniform deformation, both bulging and unbulging sections are along the path $O \rightarrow C$. After bifurcation, the bulging section follows the path $C \rightarrow D$, whereas the unbulging section takes the path $C \rightarrow G \rightarrow E$. The unbulged part expands until the whole tube-like balloon bulges up. Finally, the balloon continuously inflates following the path $E \rightarrow F$. Note that for a tube, there exists homogeneous solution as the dot line in Fig. 11(a), while for the rugbyshaped balloon $(a / b=0.01)$ the homogeneous deformation is broken initially due to the geometry imperfection.

To examine the approximation degree of rugby-shaped ellipsoids of revolution to the tube below the ratio $a / b=0.626$, the deformation characteristics of balloons with various $a / b$ are depicted in Fig. 12. We observe that the smaller the ratio $a / b$ is, the closer the deformation to the

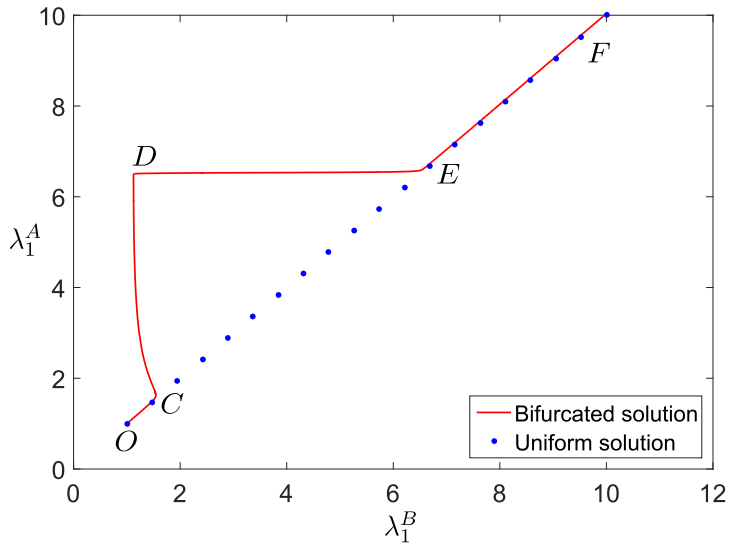

(a)

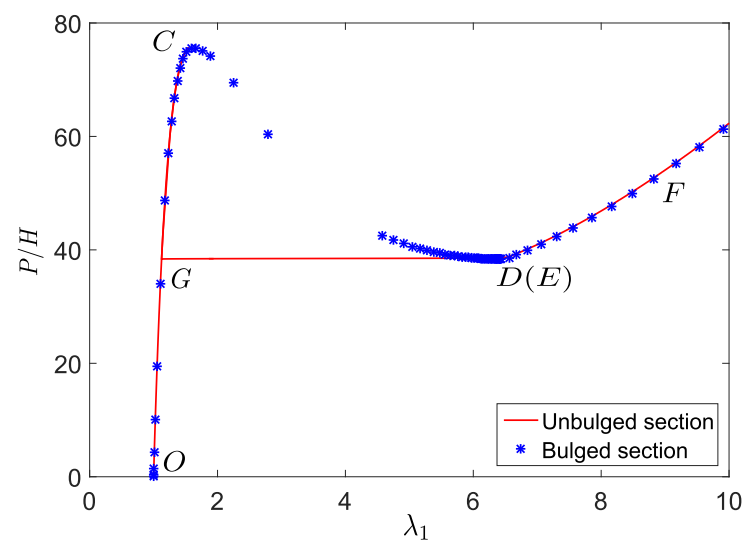

(b)

Fig. 11. Bulging and unbulging solutions of rugby-shaped balloon with $a / b=0.01$ : (a) $\lambda_{1}^{A}$ v.s. $\lambda_{1}^{B}$, where $\lambda_{1}^{A}$ corresponds to the bifurcated solution in the bulging center while $\lambda_{1}^{B}$ refers to the uniform solution away from the center. The bifurcation path follows $O C D E F$; (b) bifurcation diagram of the bulging and unbulging sections. The bifurcation curve of bulging section follows the path $O C D F$, and the bifurcation curve of unbulging section takes the path $O C G E F$.

tube is. On the contrary, the larger the ratio is, the more homogeneous the deformation is. Therefore, we can unify the balloon with the tube. The bifurcation interval for pear-shaped deformation vanishes when $a / b<0.626$. In this range, deformation of rugby-shaped balloons is analogous to that of a tube, with significant localized bulging behavior especially when $a / b<0.1$.

\subsection{Pumpkin-shaped balloon $(a / b>1)$}

For the pumpkin-shaped balloon, let us begin by setting $a / b=$ 1.25 for definiteness. The numerical primary and bifurcated $N$-shaped evolution paths are illustrated in Fig. 13, where the analytical bifurcated thresholds [22] are marked. We find that the numerical and analytical results are in good agreement. The bifurcated pear-shaped and primary spherical deformations are drawn in Fig. 14. We also examine the post-bifurcation of pumpkin-shaped balloons with different values of $a / b=1.67,2$ and 5 , etc. It appears that for any ratio $a / b>1$, the pumpkin-shaped balloon can always deform to pear shape.

\subsection{Phase diagram}

Through finite element computations of spherical, rugby-shaped and pumpkin-shaped balloons, the critical stretch $\lambda_{1}$ and the corresponding pressure $\bar{P}$ can be obtained and compared with analytical solutions, as 
Table 2

Comparison of finite element results and analytical bifurcated solutions for $\bar{P}$.

\begin{tabular}{lllll}
\hline$a / b$ & $\bar{P}_{\max }^{F E M} / \bar{P}_{\max }^{\text {Anal }}$ & $\bar{P}_{c r 1}^{\text {FEM }} / \bar{P}_{c r 1}^{\text {Anal }}$ & $\bar{P}_{c r 2}^{\text {FEM }} / \bar{P}_{c r 2}^{\text {Anal }}$ & $\bar{P}_{\min }^{\text {FEM }} / \bar{P}_{\min }^{\text {Anal }}$ \\
\hline 0.65 & $1.7079 / 1.7064$ & $1.0960 / 1.0904$ & $0.9447 / 0.9468$ & $0.7590 / 0.7582$ \\
0.8 & $1.5024 / 1.5026$ & $1.1630 / 1.1598$ & $0.8343 / 0.8369$ & $0.6577 / 0.6574$ \\
1 & $1.3025 / 1.3017$ & $1.1070 / 1.1036$ & $0.7562 / 0.7633$ & $0.5666 / 0.5663$ \\
1.25 & $1.3941 / 1.3943$ & $1.2280 / 1.2289$ & $0.8656 / 0.8697$ & $0.6094 / 0.6090$ \\
\hline
\end{tabular}

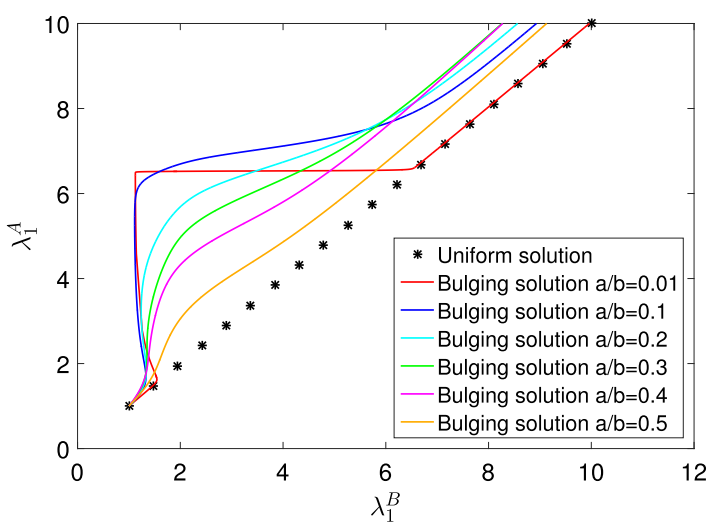

Fig. 12. Bulging solutions of rugby-shaped balloons with various $a / b$. The diagonal line represents the uniform deformation. The smaller the ratio $a / b$ is, the closer the deformation to the tube is; whereas the larger the ratio is, the more homogeneous the deformation is.

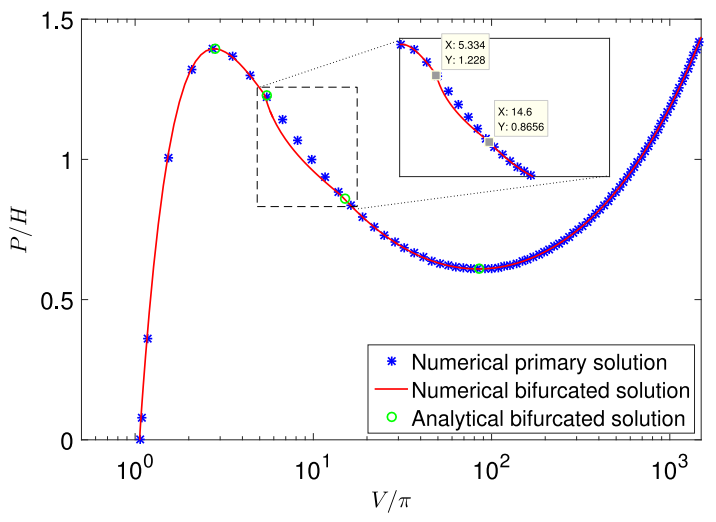

Fig. 13. Bifurcation portrait of pumpkin-shaped balloon with $a / b=1.25$. The critical bifurcated solutions are: $\bar{V}_{c r 1}=5.334, \bar{V}_{c r 2}=14.6, \bar{P}_{c r 1}=1.228$ and $\bar{P}_{c r 2}=0.8656$, where the scaling volume $\bar{V}=V / \pi$. The limit points read: $\bar{P}_{\max }=1.3941, \bar{P}_{\min }=0.6094, \bar{V}_{\max }=2.8460$ and $\bar{V}_{\text {min }}=83.6108$.

shown in Table 2 where a remarkable agreement is found. A unified phase diagram on instability mode selection of various ellipsoidal balloons is depicted in Fig. 15. When the ratio $a / b<0.626$, the rugbyshaped balloon is analogous to a tube, with localized bulging during inflation instead of pear-shaped deformation. For the other part of rugby-shaped balloons with $0.626<a / b<1$, pear-shaped deformation is usually observed. The red line refers to the spherical balloon with $a / b=1$ that has pear-shaped solutions. For pumpkin-shaped balloons $(a / b>1)$, the pear-shaped deformation with different geometries may not be exactly the same. The most important factor apart from geometry lies in the distribution of imperfection. For example, for the cases of $a / b=2$, when the perturbation is applied to the lower part of the balloon, the pear shape with upper part larger than the lower part can be obtained, and vice versa. No matter what kind of pear shapes, the common point remains that the half above and half down are not identical.

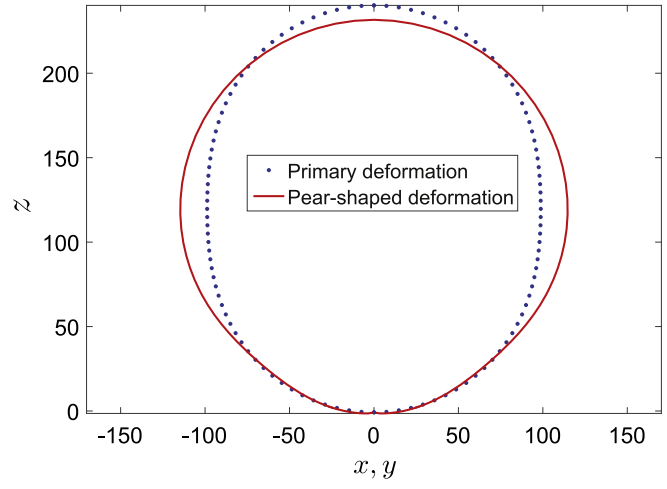

(a)

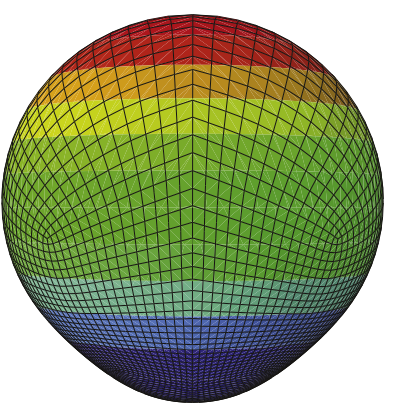

(b)

Fig. 14. Deformation of pumpkin-shaped balloon with $a / b=1.25$ within the bifurcation interval: (a) cross-section of spherical and pear-shaped deformation; (b) 3D pear-shaped deformation. Scaling factor: 100 .

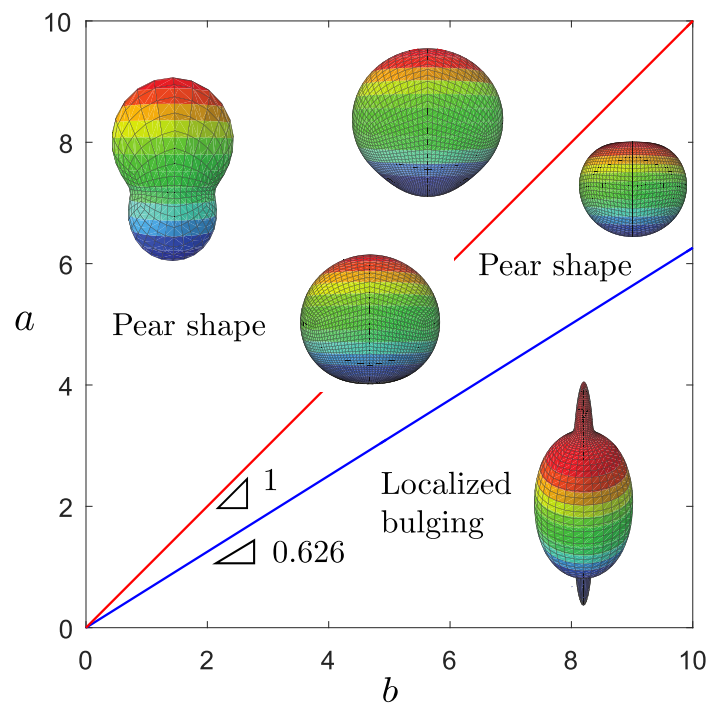

Fig. 15. Phase diagram on deformations of various ellipsoidal balloons: when $a / b>0.626$, the spherical $(a / b=1)$, pumpkin-shaped $(a / b>1)$ and part of rugby-shaped $(0.626<a / b<$ 1) balloons can bifurcate into pear shape; whereas for $a / b<0.626$, deformation turns out to be localized bulging which is usually observed in the inflation of a tube. 


\section{Concluding remarks}

Snap-through post-bifurcation evolution of various ellipsoidal balloons, including spherical, rugby-shaped and pumpkin-shaped configurations, was studied numerically. The $N$-shaped curves and the corresponding critical bifurcation points were captured, which remarkably agree with the analytical solutions in the literature. Based on finite element simulations, the effect of aspect ratio $a / b$ on the bifurcation interval was characterized quantitatively. For rugby-shaped balloons, there exists a threshold at $a / b=0.626$ to deform into pear shape. Below this value, the nonlinear behavior of the rugby-like balloon is approximated to a tube, with localized bulging instead of pear-shaped deformation. The evolution of localized bulging was first obtained numerically. Besides, the effect of various aspect ratios of the major and minor axes for rugby-shaped balloons was discussed and their nonlinear bulging responses were captured. As for pumpkin-shaped balloons $(a / b>1)$, it is always possible to bifurcate into pear shape. Lastly, a unified phase diagram on instability mode selection of various ellipsoidal balloons, including sphere, rugby, pumpkin and tube, was presented.

\section{Acknowledgments}

This work is supported by the National Natural Science Foundation of China (Grant Nos. 11602058, 11772094, 11461161008), Shanghai Education Development Foundation and Shanghai Municipal Education Commission (Shanghai Chenguang Program, Grant No. 16CG01), startup fund from Fudan University, and National Key Research and Development Program of China (2016YFB0700103). FX and MPF are also grateful for the financial support from the French National Research Agency ANR (LabEx DAMAS, Grant No. ANR-11-LABX-0008-01).

\section{References}

[1] J.G. Valdés, J. Miquel, E. Oñate, Nonlinear finite element analysis of orthotropic and prestressed membrane structures, Finite Elem. Anal. Des. 45 (2009) 395-405.

[2] J. Bonet, R.D. Wood, J. Mahaney, P. Heywood, Finite element analysis of air supported membrane structures, Comput. Methods Appl. Mech. Engrg. 190 (2000) 579-595.

[3] Y. Zhou, A. Nordmark, A. Eriksson, Multi-parametric stability investigation for thin spherical membranes filled with gas and fluid, Int. J. Nonlinear Mech. 82 (2016) $37-48$.

[4] S. Budday, G. Sommer, C. Birkl, C. Langkammer, J. Haybaeck, J. Kohnert, M. Bauer, F. Paulsen, P. Steinmann, E. Kuhl, G.A. Holzapfel, Mechanical characterization of human brain tissue, Acta Biomater. 48 (2017) 319-340.

[5] S. Rudykh, K. Bhattacharya, G. de Botton, Snap-through actuation of thick-wall electroactive balloons, Int. J. Nonlinear Mech. 47 (2012) 206-209.

[6] G. de Botton, R. Bustamante, A. Dorfmann, Axisymmetric bifurcations of thick spherical shells under inflation and compression, Int. J. Solids Struct. 50 (2013) 403-413.

[7] L. Dorfmann, R.W. Ogden, Nonlinear response of an electroelastic spherical shell, Internat. J. Engrg. Sci. 85 (2014) 163-174.

[8] R. Mangan, M. Destrade, Gent models for the inflation of spherical balloons, Int. J. Nonlinear Mech. 68 (2015) 52-58.

[9] A. Eriksson, A. Nordmark, Instability of hyper-elastic balloon-shaped space membranes under pressure loads, Comput. Methods Appl. Mech. Engrg. 237-240 (2012) $118-129$.

[10] A. Patil, A. DasGupta, Finite inflation of an initially stretched hyperelastic circular membrane, Eur. J. Mech. A-Solid 41 (2013) 28-36.

[11] A. Patil, A. Nordmark, A. Eriksson, Instabilities of wrinkled membranes with pressure loadings, J. Mech. Phys. Solids 94 (2016) 298-315.

[12] D.M. Haughton, R.W. Ogden, On the incremental equations in non-linear elasticity, J. Mech. Phys. Solids 26 (1978) 93-110.

[13] D.M. Haughton, Post-bifurcation of perfect and imperfect spherical elastic membranes, Int. J. Solids Struct. 16 (1980) 1123-1133.

[14] Y.C. Chen, T.J. Healey, Bifurcation to pear-shaped equilibria of pressurized spherical membranes, Int. J. Nonlinear Mech. 26 (1991) 279-291.

[15] I. Müller, H. Struchtrup, Inflating a rubber balloon, Math. Mech. Solids 7 (2002) 569-577.
[16] Y.B. Fu, Y.X. Xie, Stability of pear-shaped configurations bifurcated from a pressurized spherical balloon, J. Mech. Phys. Solids 68 (2014) 33-44.

[17] T. Li, C. Keplinger, R. Baumgartner, S. Bauer, W. Yang, Z. Suo, Giant voltage-induced deformation in dielectric elastomers near the verge of snap-through instability, J. Mech. Phys. Solids 61 (2012) 611-628.

[18] F. Wang, C. Yuan, T. Lu, T.J. Wang, Anomalous bulging behaviors of a dielectric elastomer balloon under internal pressure and electric actuation, J. Mech. Phys. Solids 102 (2017) 1-16.

[19] S. Qu, Z. Suo, A finite element method for dieletric elastomer transducers, Acta. Mech. Solid. Sin. 25 (2012) 459-466.

[20] E. Bortot, Analysis of multilayer electro-active spherical balloons, J. Mech. Phys. Solids 101 (2017) 250-267.

[21] X. Liang, S. Cai, Shape bifurcation of a spherical dielectric elastomer balloon under the actions of internal pressure and electric voltage, J. Appl. Mech. 82 (2015) 101002-1-101002-8.

[22] Y.N. Geng, J.X. Huang, Y.B. Fu, Shape bifurcation of a pressurized ellipsoidal balloon, Internat. J. Engrg. Sci. 101 (2016) 115-125.

[23] A.N. Gent, Elastic instabilities in rubber, Int. J. Nonlinear Mech. 40 (2005) 165-175.

[24] A. Dorfmann, D.M. Haughton, Stability and bifurcation of compressed elastic cylindrical tubes, Internat. J. Engrg. Sci. 44 (2006) 1353-1365.

[25] Y.B. Fu, S.P. Pearce, K.K. Liu, Post-bifurcation analysis of a thin-walled hyperelastic tube under inflation, Int. J. Nonlinear Mech. 43 (2008) 697-706.

[26] Y.B. Fu, Y.X. Xie, Effects of imperfections on localized bulging in inflated membrane tubes, Phil. Trans. R. Soc. A 370 (2012) 1896-1911.

[27] Y.B. Fu, J.L. Liu, G.S. Francisco, Localized bulging in an inflated cylindrical tube of arbitrary thichness-the effect of bending stiffness, J. Mech. Phys. Solids 90 (2016) $45-60$.

[28] G. Mao, T. Li, Z. Zou, S. Qu, M. Shi, Prestretch effect on snap-through instability of short-length tubular elastomeric balloons under inflation, Int. J. Solids Struct. 51 (2014) 2109-2115.

[29] J. Shi, G.F. Moita, The post-critical analysis of axisymmetric hyper-elastic membranes by the finite element method, Comput. Methods Appl. Mech. Engrg. 135 (1996) 265-281.

[30] D.C. Pamplona, P.B. Gonçalves, S.R.X. Lopes, Finite deformations of cylindrical membrane under internal pressure, Int. J. Mech. Sci. 48 (2006) 683-696.

[31] P.B. Gonçalves, D.C. Pamplona, S.R.X. Lopes, Finite deformations of an initially stressed cylindrical shell under internal pressure, Int. J. Mech. Sci. 50 (2008) 92-103.

[32] R.W. Ogden, Large deformation isotropic elasticity -on the correlation of theory and experiment for incompressible rubberlike, Proc. R. Soc. Lond. A 326 (1972) 565-584.

[33] R.W. Ogden, Non-Linear Elastic Deformations, Ellis Horwood, Chichester, England, 1984.

[34] T. Lu, L. An, J. Li, C. Yuan, T.J. Wang, Electro-mechanical coupling bifurcation and bulging propagation in a cylindrical dielectric elastomer tube, J. Mech. Phys. Solids 85 (2015) 160-175

[35] L. An, F. Wang, S. Cheng, T. Lu, T.J. Wang, Experimental investigation of the electromechanical phase transition in a dielectric elastomer tube, Smart Mater. Struct. 24 (2015) 035006-1-035006-9.

[36] E. Verron, G. Merckmann, Numerical analysis of rubber balloons, Thin Wall. Struct. 41 (2003) 731-746

[37] A.E. Giannakopoulos, A. Triantafyllou, Spherical indentation of incompressible rubber-like materials, J. Mech. Phys. Solids 55 (2007) 1196-1211.

[38] L.M. Kanner, C.O. Horgan, Elastic instabilities for strain-stiffening rubber-like spherical and cylindrical thin shells under inflation, Int. J. Nonlinear Mech. 42 (2007) 204-215.

[39] Y.X. Xie, J.C. Liu, Y.B. Fu, Bifurcation of a dielectric elastomer balloon under pressurized inflation and electric actuation, Int. J. Solids Struct. 78 (2016) 182-188.

[40] L.R.G. Treloar, Stress-strain data for vulcanised rubber under various types of deformation, Trans. Faraday Soc. 40 (1944) 59-70.

[41] ABAQUS, 2013. ABAQUS Analysis User's Manual, version 6.13.

[42] C. Ronchi, R. Iacono, P.S. Paolucci, The "cubed sphere": A new method for the solution of partial differential equations in spherical geometry, J. Comput. Phys. 124 (1996) 93-114.

[43] E.H. Boutyour, H. Zahrouni, M. Potier-Ferry, M. Boudi, Bifurcation points and bifurcated branches by an asymptotic numerical method and padé approximants, Internat. J. Numer. Methods Engrg. 60 (2004) 1987-2012.

[44] F. Xu, M. Potier-Ferry, S. Belouettar, H. Hu, Multiple bifurcations in wrinkling analysis of thin films on compliant substrates, Int. J. Nonlinear Mech. 76 (2015) 203-222.

[45] A. Eriksson, A. Nordmark, Symmetry aspects in stability investigations for thin membranes, Comput. Mech. 58 (2016) 747-767.

[46] E. Doedel, AUTO: A program for the automatic bifurcation analysis of autonomous systems, Congr. Numer. 30 (1981) 265-284.

[47] E.L. Allgower, K. Georg, Numerical Continuation Methods, Springer-Verlag, Berlin, 1990. 